Théologiques

Théologiques

\title{
Entre le devoir de pardonner et le droit de ne pas pardonner
}

\section{Karlijn Demasure et Jean-Guy Nadeau}

Volume 23, numéro 2, 2015

Théologies de la réconciliation

URI : https://id.erudit.org/iderudit/1042752ar

DOI : https://doi.org/10.7202/1042752ar

Aller au sommaire du numéro

\section{Éditeur(s)}

Faculté de théologie et de sciences des religions, Université de Montréal

\section{ISSN}

1188-7109 (imprimé)

1492-1413 (numérique)

Découvrir la revue

Citer cet article

Demasure, K. \& Nadeau, J.-G. (2015). Entre le devoir de pardonner et le droit de ne pas pardonner. Théologiques, 23(2), 253-270.

https://doi.org/10.7202/1042752ar

\section{Résumé de l'article}

Les auteurs abordent la question du pardon à partir de l'expérience des victimes d'abus sexuels qui la posent souvent d'une façon très aiguë. Le christianisme tient généralement le pardon pour un devoir chrétien, fondé sur une théologie qui considère la réconciliation comme le coeur de l'histoire de salut. Le Nouveau Testament porte un appel à pardonner qui ne peut être nié. Par contre, celui-ci ne va pas nécessairement sans condition ni en faisant abstraction du contexte de la faute ou de la relation. Opérant une étrange inversion de la dynamique du pardon, la psychologie considère de son côté les bienfaits du pardon pour celui ou celle qui le donne plus que pour celui ou celle qui le reçoit. La psychologie connaît aussi les longs cheminements et les impasses du pardon dont la théologie ne saurait faire abstraction. Cela appelle une théologie qui soit plus sensible à la justice et qui approche avec plus de retenue la question du pardon et de la réconciliation. Comme l'amour, le pardon est un don et il ne peut être forcé.

Le pardon n'est pas une obligation dont il faudrait se culpabiliser ou culpabiliser les victimes, souvent les plus faibles, de ne pas y répondre. Si la grâce de Dieu permet de pardonner là où la volonté et le pouvoir humains touchent à leurs limites, peut-être y a-t-il aussi une grâce qui permet de retenir le pardon jusqu'à ce que quelque chose change, voire une grâce qui permet de faire justice pour que la vie se poursuive en abondance. 


\title{
Entre le devoir de pardonner et le droit de ne pas pardonner
}

\author{
Karlijn Demasure" \\ Théologie \\ Université Grégorienne (Rome, Italie) \\ Jean-Guy NADEAu** \\ Théologie \\ Université Grégorienne (Rome, Italie)
}

Dans les cas d'abus sexuels à partir desquels nous avons construit notre réflexion, la question du pardon et même de la réconciliation se pose de façon très aiguë. D'un côté, on tient le pardon pour un devoir chrétien, fondé sur une théologie qui considère la réconciliation comme le cœur de l'histoire du salut. De l'autre, on est beaucoup plus réservé face au pardon en se référant tout d'abord à la psychologie ainsi qu'à une théologie de compassion et de justice qui restreint la nécessité du pardon et de la réconciliation.

* Karlijn Demasure est directrice du Centre pour la protection des mineurs à l'Université pontificale Grégorienne (Rome, Italie) où elle est également professeure. Elle a été Doyenne des Facultés de sciences humaines et de philosophie et titulaire de la Chaire de recherche sur la famille chrétienne à l'Université saint Paul d'Ottawa, Canada (2008-2014). Ses recherches actuelles portent sur l'abus sexuel. Elle a récemment publié: (2017) «Healing and the Forbidden Touch. A Reflection on Selected Scripture Stories ", dans M. Rovers, J. Malette et M. Guirguis-Younger, dir., Therapeutic Touch, Research, Practice and Ethics, University of Ottawa Press, p. 69-82.

** Jean Guy Nadeau est professeur honoraire à l'Université de Montréal et professeur invité au Centre pour la protection des mineurs de l'Université pontificale Grégorienne (Rome, Italie). Ses recherches actuelles portent sur les facteurs et modes de protection contre les abus sexuels, trauma et spiritualité, ainsi que rock et religion. Il a récemment publié: (2012) J.-G. Nadeau, C. Golding et C. Rochon, Autrement que victimes. Dieu, enfer et résistance chez les victimes d'abus sexuels, Novalis. 
Quand on a été blessé profondément, au moins deux sujets de réflexion s'imposent: la guérison de la blessure et le pardon. Personne ne se pose sérieusement la question sur la nécessité de guérir la blessure mais plutôt celle du «comment». Par contre, la question sur le besoin et le devoir de pardonner est très actuelle. La Shoah a fait surgir le doute sur le devoir de pardonner en demandant s'il existe un «mal impardonnable». Dans le temps d'après-guerre, la conviction se répandait en effet que pardonner les crimes de guerre signerait «la banalisation du mal $^{1}$ ».

Jusque-là le christianisme avait toujours soutenu la conviction que tout péché pouvait être pardonné à condition que le pécheur demande pardon, qu'il fasse pénitence et se convertisse. Dans l'argumentation, on distinguait le péché et le pécheur en affirmant que le péché ne couvre pas entièrement l'identité de la personne.

Après la Shoah, l'attention se déplace de la personne du pécheur vers la victime et sa guérison (Lascaris 1999, 49). Dès lors, la question devient très prégnante: à l'intérieur de la tradition chrétienne, est-ce que la victime doit pardonner et se réconcilier avec l'offenseur? Dans un premier temps, nous présenterons l'argumentation selon laquelle le pardon et la réconciliation sont un devoir. Dans un deuxième temps, nous exposerons le point de vue selon lequel la victime ne doit pas pardonner. Nous terminerons avec une brève réflexion sur la création des conditions du pardon.

\section{Le devoir de pardonner}

\subsection{La blessure, la mémoire et l'histoire de vie}

Pour répondre à l'appel du pardon, il faut se rappeler du mal subi. La mémoire est une condition nécessaire pour arriver à la guérison ainsi qu'au pardon. Si on ne se rappelle pas qu'il y a eu blessure, il n'y a pas de besoin de guérison (de Pisón 2011, 16-19). Cependant, il faut faire une différence entre faire mémoire et se victimiser. "Se victimiser, comme l'indique Martínez de Pisón, signifie donner le pouvoir à d'autres, abandonner le contrôle sur sa propre vie. Non seulement une telle attitude affaiblit la personne, mais elle la rend dépendante et à la merci des autres. Se souvenir

1. Le concept de la «banalité du mal» vient de Hannah Arendt qui voulait exprimer qu'un grand mal se fait souvent à partir des petites décisions administratives de chacun ou en obéissant à des ordres sans réfléchir. Il ne s'agit pas de déculpabiliser la personne puisque Arendt juge une telle attitude impardonnable. Cependant, avec l'expression de «la banalisation du mal», on réfère à une situation dans laquelle le pardon est accordé sans pour autant reconnaître l'ampleur du mal. 
est une approche active qui veille à ce que l'abus ne se répète plus. » (de Pisón 2011, 21 et 22-23). Afin que la mémoire de l'offense soit libératrice pour la personne et puisse mener au pardon, il faut cesser de s'apitoyer sur soi. Le contraire maintient la personne dans un cercle vicieux où la mémoire de la souffrance peut répéter la souffrance. En effet, en racontant l'histoire d'un événement qui nous a fait souffrir, les mêmes sentiments risquent de nous envahir de nouveau. En plus, faire mémoire d'un événement malheureux sans s'apitoyer sur son sort peut mener au pardon à soi-même, ce qui est la condition sine qua non pour pouvoir pardonner à autrui. «La haine contre soi-même pour avoir enduré tellement de sévices ressemble à un poison qui détruit la personne de l'intérieur. " (de Pisón 2011, 20) La mémoire est donc nécessaire au pardon, mais la question qui se pose est celle de savoir comment l'utiliser pour qu'elle y mène. Pour répondre à cette question, il faut réfléchir au rôle de la mémoire dans la construction de l'identité.

La mémoire crée l'identité parce que sans notre mémoire nous ne savons plus qui nous sommes. C'est là l'horreur de la démence. On pourrait objecter qu'on reste un individu grâce à la mémoire de l'autre qui chérit les souvenirs d'une vie partagée, mais la conscience de soi est irremplaçable et se trouve perdue à jamais si la mémoire est tout à fait éliminée. La mémoire est nécessaire pour la construction de l'identité ${ }^{2}$, ce qui se fait dans l'histoire de vie. La mémoire s'exprime dans les récits que nous racontons sur nous-mêmes. La personne choisit entre de nombreux événements qui sont arrivés au cours de sa vie: entre les faits fortunés ou douloureux, entre les sentiments de joie ou de tristesse et entre les actes bons ou les actes dont elle a souffert. Tous les événements ne peuvent faire partie de l'histoire de vie, c'est l'individu qui en fait le choix. C'est la liberté du «quoi » du récit. On peut conclure que la mémoire crée l'identité mais dans une dynamique dialectique, l'être humain crée aussi la mémoire, parce qu'il choisit les éléments qui font partie de son récit de vie et qu'il n'oubliera pas. Avec le choix, se tient aussi la responsabilité, une responsabilité par ailleurs limitée par les moyens psychologiques et langagiers à notre disposition. On peut construire une histoire de vie qui renforce la tragédie de l'abus ou on peut raconter l'histoire en fonction de l'espoir d'être guéri.

On pourrait objecter que la mémoire est bien plus que ce qui s'exprime dans les récits partagés. Il y a des événements, des souvenirs que nous ne racontons jamais et qui cependant marquent notre identité profondément.

2. Voir Comblin (1991); Ricœur (1983). 
En plus de ces récits passés sous silence, il y a ces images sans paroles qui souvent nous hantent et qui cherchent à s'exprimer. Elles aussi font partie de notre identité. Souvent ce sont des images des événements qui nous ont touchés d'une façon très profonde: des traumas, par exemple l'abus sexuel jamais raconté. La psychologie nous a appris que la guérison n'est possible que lorsque les souvenirs s'expriment dans les paroles. Il en est de même pour le pardon. Il n'y a pas de pardon ou de réconciliation possible sans paroles: la blessure doit être exprimée, comme est aussi nécessaire la confession de l'offenseur.

\subsection{Histoire de vie et identité chrétienne}

L'identité se construit en intégrant les événements de bonheur et de malheur dans notre récit de vie. Mais cette construction ne se fait pas dans le vide. Dans la culture, des master stories circulent, influencent et sont parties prenantes de notre histoire de vie. Pensons, par exemple, aux récits patriarcaux ou aux récits idéologiques qui marquent notre culture. Souvent cette intégration se fait inconsciemment, mais elle peut aussi être objet de choix. Le christianisme est une telle master story et si on veut se construire une identité chrétienne, l'histoire du Christ doit faire partie de notre histoire de vie. Chrétiens et chrétiennes doivent donc intégrer l'histoire du salut dans leur récit de vie. L'histoire de vie de Jésus Christ et celle de notre propre vie s'entrelacent et notre passé se trouve racheté dans cet entrelacement. Or le christianisme a aussi une master story sur le pardon, selon laquelle il faut pardonner soixante-dix fois sept fois, aimer son ennemi, présenter l'autre joue, etc. Comment peut-on interpréter cela?

\subsection{La Memoria Christi et la rédemption de tous}

«Beaucoup de victimes croient qu'elles n'ont pas d'obligation à pardonner et à se réconcilier avec l'abuseur. Elles sont convaincues qu'en aimant l'abuseur, elles ne réalisent pas l'humanité mais la trahissent plutôt. (Volf 2006, 9)

Dès l'Ancien et le Nouveau Testament, le pardon est une dimension constitutive de l'histoire du salut. Comme en témoignent parmi d'autres Os 11,7-9; Mi 7,18-20; Is 54,4-10; Ps 30,6, Dieu vient constamment «à la rencontre de son peuple infidèle pour lui offrir à nouveau son pardon et son amour.» (de Pisón 2000, 91, voir aussi 92-98) Le pardon divin se révèle dans toute sa plénitude en Jésus Christ et il se manifeste de façon 
particulière dans la parabole de l'enfant prodigue (Lc 15,11-32; voir Duquoc 1986, 49). Ainsi, "[1]e pardon divin, représenté [par cette parabole], dépasse toute compréhension et toute logique humaines: il est le fruit de la gratuité divine, parce qu'il n'est conditionné par rien.» (de Pisón, 2000, 95-96) Jean-Paul II l'exprimait clairement dans la Lettre encyclique sur la miséricorde divine (Jean-Paul II 1980, $\mathrm{n}^{\text {os }} 4.12 .14$ ). Sans la miséricorde, voire sans le pardon, la justice peut devenir une façon voilée de se venger ${ }^{3}$.

Mais allons directement au cœur de la foi chrétienne. Partons de l'eucharistie qui est au centre de la vie chrétienne et dans laquelle nous célébrons, nous commémorons la mort et la résurrection de Jésus Christ. Nous faisons cela en mémoire de lui. Le narratif de la passion et de la résurrection est intensément lié au récit de l'Exode. Rappelons-nous que la Cène avait lieu le jour où les juifs commémorent l'Exode. Or l'Exode jette la lumière sur plusieurs caractéristiques de Dieu:

- Dieu a sauvé son peuple souffrant en le délivrant de l'esclavage.

- Il a puni les Égyptiens pour avoir causé cette souffrance, comme le raconte l'histoire des plaies d'Égypte dont la fonction de châtiment paraît alors s'ajouter à celle de signe ou de moyen de pression.

- Il a mené son peuple vers la terre promise.

Cette théologie est libératrice pour la victime d'abus sexuels ou d'autres blessures: la libération de la souffrance infligée injustement reflète un Dieu sauveur des victimes. Mais c'est aussi un Dieu qui punit les abuseurs et se manifeste ainsi comme un Dieu juste. Enfin, c'est la promesse d'une vie meilleure qui le caractérise fondamentalement comme le Dieu libérateur. Il mettra fin à la souffrance des victimes d'abus même si le chemin vers la terre promise est long.

La théologie de la libération a fortement insisté sur Dieu qui se positionne du côté des souffrants. Elle voit la même dynamique dans la mort et la résurrection du Christ. En Christ, Dieu se positionne définitivement du côté des victimes. Son corps crucifié, torturé, couvert de plaies et mis à mort est le sujet de beaucoup de tableaux. Ce corps injustement abusé

3. «Jésus Christ révèle l'origine divine du pardon de Dieu qui est au-dessus de la seule justice, qu'il ne supprime pas. Mais la justice sans la miséricorde est froide et peut devenir, tout simplement, une vengeance voilée. La justice de Dieu est aussi une manifestation de son amour et de sa miséricorde; elle est une justice qui libère, parce qu'elle nous justifie devant Dieu (voir Ps 40,11; 98,2-3; Is 45,21; 51,5. 8; 56,1)» (de Pisón 2000, 98). 
permet une identification aux personnes qui ont souffert d'abus sexuels. Le corps abusé de Jésus fait image de leur propre corps ${ }^{4}$. Il leur parle dans un langage qui ne peut être égalé par des paroles, même si elles ont été choisies avec un soin incomparable. Les mots désespérés de Jésus: "Mon Dieu, pourquoi m'avez-vous abandonné ? " font écho aux soupirs les plus profonds des victimes. La résurrection indique que non seulement notre âme mais aussi nos corps sont sanctifiés après avoir été désacralisés.

L'incorporation de la souffrance, de la mort et de la résurrection de Jésus Christ dans sa propre histoire de vie crée une nouvelle identité pour la victime. Souvent, ce que la victime a vécu semble constituer toute son identité aussi bien pour elle-même que pour les gens autour d'elle: elle est une survivante de l'holocauste, elle est une victime d'abus sexuels. Mais le christianisme considère que la personne est définie au niveau le plus profond par Dieu et par son amour envers nous, qu'importe ce qui nous arrive (Volf 2006, 78-79). Il est bien possible que des malheurs nous arrivent et qu'ils nous semblent dépourvus de tout sens; mais chaque fois qu'on donne un sens positif à un mal souffert, on le fait dans l'espoir qu'à la fin des temps, il y aura révélation du sens complet.

Miroslav Volf nous amène sur une autre piste. Dans son livre The End of Memory. Remembering Rightly in a Violent World, Volf juge cette interprétation de la théologie de la libération incomplète parce qu'elle ne va pas au-delà de l'interprétation de l'Exode. Dans ce cas-là, le récit de la passion du Christ n'ajouterait rien de nouveau. Pour lui, la passion et la résurrection du Christ ajoutent des éléments fondamentaux au récit de l'Exode. On passe d'un Dieu juste à un Dieu qui dépasse cette justice en offrant la grâce: c'est le scandale de la croix.

Dans le récit de la passion, il s'agit d'une rédemption qui va au-delà d'un seul peuple. Le passage du peuple d'Israël à tout peuple signifie plus qu'un changement quantitatif. Il implique le peuple d'Égypte, il implique les abuseurs. Le récit s'oppose indirectement à une punition qui fait souffrir tout un peuple sans tenir compte de la culpabilité ou de l'innocence individuelle. Les commandements qui sont inscrits dans le récit de l'Exode sont remplacés par le commandement d'aimer l'autre comme soi-même, l'ennemi inclus. À la théologie de la libération, qui met au centre le couple souffrance/délivrance, il faut ajouter le couple ennemi/réconciliation. En

4. Malgré la difficulté fréquente des femmes à s'identifier au corps masculin du Christ, la pratique clinique et pastorale indique que, dans les cas d'abus sexuels, les femmes aussi bien que les hommes y retrouvent leur expérience. 
effet, le Christ n'est pas seulement mort pour ceux qui souffrent, il est mort aussi pour ceux qui ont péché. Il a réconcilié les pécheurs avec Dieu et les êtres humains les uns avec les autres.

Les deux récits, notamment celui de l'Exode et celui de la passion/ résurrection, sont pour Volf des récits de rédemption. Il faut les considérer comme des méta-mémoires (Volf 2006, 94) qui nous fournissent des structures pour intégrer nos souvenirs. En tissant le récit de la passion dans la toile qu'est son récit de vie, le chrétien se sait appelé à se réconcilier avec son offenseur. Ces méta-mémoires sacrées ont le don de jeter un pont entre le passé et le futur. Cela signifie, pour la victime qui est en mesure de pardonner et de se réconcilier à l'exemple du Christ, que l'abus ne sera plus au centre de son identité et que la mémoire de l'abus ne témoignera que du passé, sans être déterminante pour le présent ou pour le futur. La réconciliation guérit et crée l'espoir pour le futur sans pour autant nier la réalité des injustices subies (Volf 2006, 121). Bien sûr, l'offenseur peut refuser le don du pardon, mais cela ne peut pas mener à ce que ce don soit retiré parce que le don ne connaît pas de conditions; il est gratuit. Mais le refus de l'acceptation du don mène à une suspension du pardon.

Revenons à l'eucharistie. Dans l'eucharistie nous commémorons les méta-récits de l'Exode et de la Passion. Dans cette communauté qui s'unit au Christ, nous nous souvenons qu'il nous a réconciliés avec Dieu et par conséquent nous sommes réconciliés les uns avec les autres. Les métamémoires de l'Exode et de la Passion nous fournissent donc la réponse à la question formulée au début: comment se souvenir? La mémoire de la blessure doit être située à l'intérieur du cadre de la réconciliation.

\subsection{Conclusion intérimaire}

Rappelons brièvement l'argument qui considère la réconciliation comme un devoir chrétien. Si quelqu'un veut s'identifier comme chrétienne ou chrétien, l'histoire du salut devra faire partie de son histoire de vie. Le point central de l'histoire du salut, c'est la réconciliation qui s'est faite par le Christ sur la croix. Ce récit dépasse le récit de l'Exode. La théologie de la libération a insisté sur le Christ qui souffre avec les souffrants qui peuvent ressusciter avec lui. Mais ceci n'est que la moitié de la bonne nouvelle. Il faut ajouter l'élément qui est aussi important que le premier: le Christ a aussi réconcilié les pécheurs avec Dieu. Il n’y a pas d'exception; la rédemption s'adresse à tous et à toutes. La résurrection nous 
rappelle que la réconciliation est voulue par Dieu et nous indique que nous pouvons vivre dans l'espoir d'un monde meilleur. Par la mort et la résurrection de Jésus Christ, chaque chrétien est appelé non seulement à aider les souffrants à se libérer de la souffrance mais aussi à se réconcilier avec son ennemi. Voilà la première perspective que nous voulions présenter.

\section{Le droit de ne pas pardonner}

\subsection{La différence entre le pardon et la réconciliation}

La seconde perspective de notre propos est opposée à la première. Elle distingue d'abord pardon et réconciliation. Le pardon est un processus intérieur, alors que la réconciliation implique aussi une relation avec l'agresseur et des démarches de sa part. Pardon et réconciliation peuvent aller de pair, mais dans un monde qui considère que c'est la victime qui bénéficie du pardon qu'elle donne, le pardon ne saurait attendre le bon vouloir de l'offenseur, ce qui serait encore lier la victime au pouvoir de son agresseur. Il est possible que celui-ci ne reconnaisse pas le mal qu'il a fait, possible qu'il ne veuille pas du pardon offert. Il y aurait alors pardon mais pas réconciliation. Si on se réconcilie, c'est pour que ça aille mieux ou pour retrouver la relation qui existait auparavant. En certains cas, la relation sera positive, sans nécessairement retrouver sa qualité antérieure. En d'autres cas, plus malheureux mais bien documentés, elle redeviendra une relation de domination, ce qui fera par la suite partie des raisons de refuser non seulement de se réconcilier, mais bien de refuser de pardonner.

Enfin, la réconciliation n'est possible que si le pardon qui la précède n'est pas un devoir. Sinon, il y a un de ses acteurs qui s'y trouve forcé, encore une fois. Dans notre culture, c'est sur la victime que pèse alors le poids de la réconciliation car c'est à elle qu'on demande de pardonner, de remettre les dettes, dettes morales en ce cas.

\subsection{Un embarras: pardonner et remettre les dettes}

L'Évangile utilise trois termes pour parler du pardon: aphiemi, charizomai, et apoluo. Ces trois mots - surtout aphiemi, qui est le plus fréquent pour parler du pardon interpersonnel — sont aussi utilisés pour parler des dettes ou de la libération d'un prisonnier. Keene (1995) considère qu'il s'agit là d'actes financiers ou politiques dont la capacité n'appartient qu'aux puissants de la société. Il n'y a pas d'instance, poursuit-il, où une personne est 
pardonnée par quelqu'un de plus bas dans la hiérarchie du pouvoir, aucun exemple où le plus faible doive pardonner au plus fort. Même Jésus sur la croix ne pardonne pas à ses bourreaux; il demande au Père de le faire.

Dans l'Évangile, le pardon ou plus précisément la remise des dettes va de celui qui a vers celui qui n'a pas; elle va du puissant vers le faible, qui risque de tout perdre ou d'être vendu en esclavage s'il ne rembourse pas sa dette (Keene 1995). Le pardon va de Dieu vers les hommes et les femmes qu'il pourrait annihiler ou soumettre au feu éternel. Il nous semble que c'est sur ce fond de scène de la puissance qu'il faut comprendre la parole que Jésus en croix adresse à son Père: «Père pardonne-leur car ils ne savent pas ce qu'ils font. » Pour la mentalité religieuse, ces gens viennent de tuer le Fils de Dieu, le fils du Seigneur le plus puissant! Leur vie est en jeu, comme celle des vignerons de la parabole qui ont tué le fils de leur maître et que ce dernier exterminera à son arrivée (Mt 21). On voit clairement la valeur du pardon qui va du plus fort vers le plus faible, une valeur qu'illustre a contrario la finale du magnifique film Dogville de Lars von Trier (von Trier 2003).

Ce qui est embarrassant aujourd'hui, c'est qu'on demande au plus faible de pardonner au plus fort. En plus, le pardon dans le discours quotidien, surtout dans le discours psycho-thérapeutique, et peut-être même dans le discours politique, vise le bien de celui qui pardonne... et non le bien de celui qui est pardonné. On ne pardonne plus pour le bien ou le salut de l'autre, pour lui sauver la vie. On pardonne pour soi-même, parce que ça fait du bien, parce que ça libère celui qui pardonne! Notons tout de même que cette orientation de pardonner pour son propre bénéfice se trouve déjà dans l'Évangile: «remets-nous nos dettes comme nous remettons les dettes", «pardonnez-nous comme nous pardonnons». À moins qu'on ne lise ces textes comme suggérant que Dieu peut bien nous pardonner puisque nous aussi nous pardonnons. Matthieu 7,2 affirme qu'on sera jugé avec la même mesure avec laquelle on aura jugé, mais il est alors question de jugement et non de pardon.

D'où le malaise de notre question initiale. Si l'on saisit le pardon en fonction du bien de la victime, celle-ci doit-elle pardonner pour son bien ou y résister pour son bien?

\subsection{Refuser de pardonner}

Mais pourquoi refuser de pardonner? Pourquoi faire valoir le droit de ne pas pardonner si le pardon est si noble? Nous avancerons cinq raisons qui 
procèdent non de définitions théoriques du pardon mais de la perspective des victimes à qui on demande de pardonner. Refuser de pardonner:

- pour se protéger

- pour protéger ses valeurs, pour refuser le mal, pour sauver la justice - pour résister à la vision du monde de l'agresseur

- pour ne pas accroître le pouvoir du plus puissant

- parce que certaines demandes de pardon ont quelque chose d'indécent.

\subsubsection{Refuser de pardonner pour se protéger}

Chacun peut se rappeler des offenses, des coups et des blessures qu'il n'a pas encore pardonnés ou encore des pardons sur lesquels il a longtemps hésité. Et d'abord pour se protéger en se gardant à distance de l'agresseur. Car tout se passe bien souvent comme si la proximité avec l'offenseur restait dangereuse... d'autant que certains considèrent le pardon comme une permission de recommencer. C'est une situation que connaissent bien les femmes victimes de violence conjugale. Dans ce cas, plus les gens pardonnent, plus on peut les frapper de nouveau. Le pardon est parfois reçu comme une permission de faire le mal et on comprend que certains craignent qu'il ne le soit. Une des personnes que nous avons interviewées dans le cadre de notre recherche sur les victimes d'abus sexuels nous avait dit: "Je n'avais plus peur, alors j'ai pardonné rapidement.» (Nadeau, Golding et Rochon 2012, 149)

Il est d'autant plus difficile de pardonner qu'on s'en est parfois voulu de l'avoir fait. On a alors eu l'impression de s'être fait avoir, que l'autre en a profité alors qu'on croyait — qu'on espérait — qu'il pouvait s'amender et changer. Ainsi hésite-t-on à pardonner. Non seulement à cause de la vivacité de la blessure ou à cause de la colère, mais de peur que l'autre n'en profite, de peur de se faire prendre encore une fois, d'y perdre de nouveau sa dignité, de peur de tolérer et d'encourager l'injustice. Ce risque que la victime soit à nouveau abusée à travers le pardon lui-même explique en bonne partie le soupçon avec lequel la théologie féministe ou la littérature sur les abus sexuels abordent la question du pardon.

\subsubsection{Refuser de pardonner pour protéger ses valeurs, refuser le mal, sauver la justice}

Il y a des cas où pardonner serait nier sa propre dignité, accepter le mal et l'injustice, voire s'en faire complice. Certains pardons apparaissent comme 
injustes. Dans ces cas, ne pas pardonner, c'est refuser de se transformer en paillasson, et on sait combien les théologiennes féministes ont dénoncé une théologie de soumission (doormat theology).

Jeffrie Murphy considère que le ressentiment n'est pas toujours négatif (McKnight 2004). Non pas que les victimes soient obligées de le ressentir, mais il est pour certains un signe de respect de soi. McKnight rappelle la question de Murphy trop rarement abordée et dans laquelle nous nous reconnaissons tout à fait :

$[\mathrm{H}]$ ow far one can go in the direction of forgiveness without compromising values of genuine importance? [...] How does one maintain self-respect, selfdefence, and (especially) the moral order when one forgives? What happens to self-respect and the moral order when humans set up a strategy of pursuing forgiveness? Will wrongdoers repent? Will they learn? (McKnight 2004)

\subsubsection{Refuser de pardonner pour résister à la vision du monde,} à l'idéologie de l'agresseur

Il y a des cas où l'offenseur demande pardon mais ne change rien à son comportement ou n'en prend pas les moyens. Certains agresseurs sollicitent même le pardon alors qu'ils continuent les abus:

Mon père venait dans ma chambre la nuit pour me toucher. Avant de partir, il exigeait que je lui pardonne. Il me disait que si j'en parlais à qui que ce soit, même lorsque je serais adulte, ça voulait dire que je ne lui avais pas vraiment pardonné. J'irais en enfer parce que Dieu ne me pardonnerait pas ${ }^{5}$.

Dans une telle situation, pardonner signifierait ainsi garder le silence, laisser faire! La maxime biblique est alors utilisée par l'adulte pour contraindre l'enfant au silence, ce qui constitue une caractéristique des abus sexuels contre les enfants (voir Nadeau 2001).

On dira qu'il ne s'agit que d'un stratagème, que d'une perversion d'une maxime biblique. Mais qui apprend à l'enfant de six ou huit ans à faire la distinction? Ce qu'on apprend plutôt à l'enfant, c'est qu'elle doit pardonner et que le pardon divin, c'est-à-dire son salut éternel, dépend de ce pardon. Le remords de l'agresseur peut bien apparaître sincère, - ce en

5. «My dad would come into my room and fondle me at night. Before he'd even leave, he would demand that I forgive him. He said that if I ever told anyone, even when I was an adult, it meant that I hadn't really forgiven him. I would go to hell because God wouldn't forgive me.» (Heggen 1993, 96). 
quoi excellent les manipulateurs — alors que la victime croit qu'elle n'agirait pas de façon chrétienne si elle rapportait ou dénonçait l'abus. Après tout, ne doit-elle pas pardonner? Tout se passe trop souvent comme si le pardon n'avait qu'une facette, celle de l'obligation.

\subsubsection{Refuser de pardonner pour ne pas accroître le pouvoir du plus puissant}

Ce n'est pas par désir de vengeance que certains refusent de pardonner mais par souci de justice. C'est comme si, pour eux, pardonner accroîtrait le pouvoir du plus puissant, sur les autres et pas seulement sur celui qui refuse de pardonner. On pourrait peut-être relire dans cette perspective la parabole du serviteur impitoyable à qui le maître remet sa dette ou pardonne, en Matthieu 18,21-35. S'il faut pardonner jusqu'à soixante-dix fois sept fois (Mt 18,23), le maître, lui, ne pardonne pourtant qu'une seule fois, comme pour empêcher le serviteur impitoyable de faire encore du tort. Dans ce cas, le pardon est juridique, mais c'est bien l'exemple que l'Évangile en donne. L'Évangile ne paraît pas demander de tout pardonner tout le temps, comme l'indiquent aussi d'autres paraboles comme celles des vignerons homicides (Mt 21), du Jugement dernier (Mt 25), ou même celle de l'homme qui n'a pas le bon vêtement pour les noces (Lc 14), celle des talents (Lc 19). Autant de gens durement jugés, exécutés ou voués à la Géhenne. Tout se passe comme si le Maître, quel qu'il soit, ne pardonnait pas toujours. En témoignent encore les propos tranchants de Luc 15, 1-7 qui vouent au feu éternel les pécheurs qui ne font pas ce qu'il faut pour se libérer du péché. L'Évangile n'est pas toujours facile à saisir et il y a place à en discuter. Cette question du pouvoir est très importante, particulièrement dans les cas d'abus de pouvoir — et nous y reviendrons.

2.3.5 Refuser de pardonner parce que certaines demandes de pardon ont quelque chose d'indécent

Parfois, on se demande pourquoi telle ou telle demande de pardon est faite. Qu'est-ce qui est visé au juste ? Pour les intérêts de qui ? Le pardon est contextuel, marqué par la situation de ceux qui le demandent, l'accordent ou le refusent. Il est des cas où la demande de pardon a quelque chose d'indécent, soit parce que l'expression des regrets sonne faux, soit - comme on l'a dit plus haut - parce que les actes se poursuivent. On 
a même vu des abuseurs sexuels prier avec leur victime après les actes pour demander que Dieu leur pardonne (Nadeau, Golding et Rochon 2012, 87). On voit combien cette demande est perverse alors que le coupable inclut la victime dans la responsabilité et la culpabilité de ses actes. Il y a d'autres cas où l'expression des circonstances atténuantes occupe plus de place que la reconnaissance des torts qui ont été faits. Il est des cas, par exemple quand des officiers d'églises ou de compagnies commerciales demandent pardon, où l'on sent que c'est simplement en vue de se refaire une image, de retrouver un pouvoir que l'opinion publique a mis à mal ou pour faire oublier le mal qui a été fait. Il est des cas où celui qui fait la demande ne s'engage dans aucune réparation ou aucune réparation significative. Dans de tels cas, il est clair que le bien de la victime n'est pas visé, qu'il n'a pas d'intérêt pour celui qui demande pardon. On sent alors que la demande a quelque chose de grossier, de choquant, et elle doit probablement même être refusée. Encore une fois, le pardon n'est pas un dû.

\section{Créer les conditions pour un pardon}

Voyons quelques autres conditions du pardon.

\section{1 Éviter le pardon rapide}

Les victimes sont souvent mises sous pression pour pardonner. Marie Fortune $^{6}$, théologienne et pasteure américaine, fondatrice en 1987 du Center for the Prevention of Sexual and Domestic Violence et plus récemment du Journal of Religion and Abuse, travaille depuis plusieurs années avec des victimes d'abus sexuels. Elle note qu'un pardon trop rapide engendre un risque de re-victimisation, qu'il retarde ou bloque le processus de guérison, même à l'âge adulte. En effet, la famille et le clergé commencent trop souvent leur intervention en incitant la victime à pardonner. Si la victime en est incapable, elle se culpabilise et croit que Dieu ne lui pardonnera pas non plus. Le pardon est sans doute crucial au processus de guérison, mais il ne peut ni ne doit être hâté.

Pour que le pardon soit viable, voire constructif, il faut que les victimes soient prêtes à pardonner et un long processus de guérison, de thérapie précède souvent ce moment. Dans son livre Comment pardonner? Jean

6. Voir Fortune $(1988 ; 1987)$; Heggen $(1993,123)$. 
Monbourquette décrit très bien les étapes psychologiques à franchir avant d'être capable de pardonner et ce processus n'est pas même une garantie que la personne puisse pardonner. Le pardon n'est pas seulement une question de psychologie ni même de théologie; il faut aussi la grâce pour y parvenir.

\subsection{La nécessité d'un discours de vérité}

L'offenseur doit reconnaitre sa faute s'il veut obtenir le pardon, au sens classique du terme où le pardon était fait pour le bien de l'offenseur. La situation est différente quand le pardon vise le bien de celui ou de celle qui pardonne. On ne saurait alors lier sa libération à la bonne volonté de l'offenseur et donc à ses aveux. Par contre, la reconnaissance de la faute et du mal commis est nécessaire si on souhaite la réconciliation.

Si le mal commis a été le fait d'une institution, Margaret Arms insiste pour que la vérité soit dite par l'institution concernée (Arms 2002). Pour l'abus sexuel par le clergé, c'est l'Église qui doit avouer le tort fait. Arms met en garde contre un pardon individuel lorsque l'institution n'admet pas l'abus. Si la vérité de l'abus sexuel par le clergé n'est pas reconnue par la paroisse, le diocèse ou le magistère, on risque de soigner les blessures d'une façon superficielle. De nos jours, on constate que la vérité quant à ces abus est reconnue par le pape, mais dans beaucoup d'églises locales, il y a toujours le déni ou le silence. Comment peut-on offrir le pardon à un prêtre si dans le discours public toute responsabilité est niée? Arms est d'avis que le pardon ne peut «durer» et ne pas être regretté que si celui qui le donne est supporté par une communauté qui admet le mal qui a été fait (Arms 2002, 113).

Comprendre clairement la réalité complexe de l'abus et de ses conséquences est nécessaire avant d'envisager le pardon. Cette vérité, qui peut être considérée comme un acte de résistance contre le mal, se dit aussi au tribunal. C'est un des endroits où la victime peut être écoutée. Le pardon n'exclut pas la justice! Au contraire, on pourrait dire que la justice doit précéder le pardon. Cependant, justice faite ne mène pas nécessairement au pardon. On peut aller au tribunal sans avoir l'intention de pardonner. En plus, la justice rétributive peut fixer l'offenseur dans une position où il prétend violemment qu'il n'a pas commis le délit ou que ce n'était pas de sa faute. Une telle attitude ne favorise pas le pardon de la part de la victime. Seule la justice qui contribue à l'empowerment de la victime peut privilégier le pardon et il est des cas où la justice réparatrice peut le faire mieux que la justice rétributive. 


\subsection{Le pouvoir comme condition du pardon}

Marie Fortune a souvent développé l'idée que la justice constitue une des conditions de la guérison et du pardon. D’où la nécessité de rendre justice (making justice) à la victime, que ce soit de la part de l'agresseur, de l'entourage de la victime ou de la communauté. En fait, l'expérience de justice contribue à rétablir la valeur et le pouvoir de la victime. Justice as empowerment, pourrions-nous dire. Ce n'est qu'à la condition que soit restauré le pouvoir de la victime que le pardon peut devenir une option réelle... et non un nouveau fardeau. Le pardon (au sens classique) ou la réconciliation ne devient possible que lorsque le plus fort abandonne sa position dominante en se repentant sincèrement et en faisant restitution. C'est pourquoi la reconstruction du pouvoir de la victime nié dans l'abus doit précéder le pardon.

Dans cette perspective d'empowerment, il est important de considérer que le pardon n'est ni un dû, ni un droit, ni une dette de la victime envers l'agresseur. Certes, il peut être appelé par les pardons que l'on a soi-même reçus, mais il reste un don, un don à l'autre et à soi. "Pardon demandé n'est pas pardon dû », considère Paul Ricœur $(1996,81)$ qui s'est longuement penché sur la question dans La mémoire, l'histoire, l'oubli' ${ }^{7}$; sinon, quel en serait le don? La victime a le droit de ne pas pardonner.

Demander pardon, quand cela est fait sérieusement, c'est modifier sa relation avec la victime. C'est se dire dépendant de l'autre alors qu'il était auparavant en position de domination face à elle. Désormais, c'est la victime qui peut exercer du pouvoir sur la relation. Demander pardon sérieusement, c'est reconnaître à la victime le pouvoir de pardonner — ou de ne pas pardonner. C'est lui reconnaître un pouvoir que l'offense, justement, a nié, a brimé, et dont elle a bien besoin si elle veut pardonner.

\section{Conclusion}

Le Nouveau Testament porte un appel à pardonner qui ne peut être nié. Cependant, le pardon n'est pas une obligation dont il faudrait se culpabiliser de ne pas y répondre. On manque certes des occasions de pardonner, et c'est dommage. Mais il arrive que l'on en soit incapable, empêtré dans nos blessures et nos émotions ou parce que les conditions ne sont pas les bonnes. Serions-nous alors coupables de ne pas pardonner? Non! On ne

7. Ricœur (2000); voir aussi Ricœur (1990). 
saurait oublier la psychologie lorsqu'on parle de théologie, ni que l'évangile n'est pas en tout ceci sans nuance.

On peut même aller jusqu'à considérer que pardonner n'est pas un devoir moral. Comme l'amour, le pardon est un don qui ne peut être forcé. Stanislas Breton considère qu' 'il ne peut y avoir pardon que sous une loi de surabondance» (Breton 1993, 106). Dans le christianisme, c'est la grâce de Dieu qui permet de pardonner là où la volonté et le pouvoir humains touchent à leurs limites. Et peut-être y a-t-il même une grâce qui permet de retenir le pardon jusqu'à ce que quelque chose change, voire une grâce qui permet de faire justice pour que la vie se poursuive en abondance.

\section{Références}

Arms, M. F. (2002), "When Forgiveness is not the Issue ", Journal of Religion \& Abuse, 4/4, p. 107-128.

Breton, S. (1993), «L'Autrement du monde ", dans O. Abel, dir., Le pardon. Briser la dette et l'oubli, Paris, Éditions Autrement.

Comblin, J. (1991), Anthropologie chrétienne / trad. par R. Paratte, Paris, Cerf (Libération).

Duquoc, C. (1986), «Le pardon de Dieu», Concilium, 204, p. 49-58.

De Pisón, R. M. (2000), Le péché et le mal, Montréal, Médiaspaul (Brèches théologiques 32).

(2011), «Enrichie par la guérison », Counseling et spiritualité, 30/1, p. 13-28.

Fortune, M. M. (1987), «Making Justice. Sources of Healing for Incest Survivors ", Woman of Power, <http://www.faithtrustinstitute.org/ resources/articles/Making-Justice.pdf>, consulté le 22 juin 2016.

(1988), «Forgiveness. The Last Step ", dans A. L. Horton et J. A. Williams, dir., Abuse and Religion, Lexington / Toronto, Lexington Books, p. 215-220.

Heggen, C. H. (1993), Sexual Abuse in Christian Homes and Churches, Scottdate, Herald Press.

Keene, F. (1995), «The Politics of Forgiveness. How the Christian Church Guilt-Trips Survivors ", On the Issues, <http://www.caic.org.au/ biblebase/abuse/guiltabuse.html>, consulté le 22 juin 2016.

Jean-Paul II (1980), Lettre encyclique sur la miséricorde divine (Dives in misericordia), Montréal, Fides (L'Église aux quatre vents). 
LASCARIs, A. (1999), «Kan God vergeven als het slachtoffer niet vergeeft », Tijdschrift voor Theologie, 39/1, p. 48-68.

McKnight, S. (2004), «Slowing Down the Runaway Forgiveness Truck. Is there such a thing as too much mercy? ", <http://www.christianitytoday. com/bc/2004/004/19.36.html>, consulté le 22 juin 2016.

Nadeau, J.-G. (2001), «Les abus sexuels, un drame spirituel », Sciences pastorales, 20/2, p. 207-229.

Nadeau, J.-G., C. Golding et C. Rochon (2012), Autrement que victimes. Dieu, enfer et résistance chez les victimes d'abus sexuels, Montréal, Novalis.

Ricceur, P. (1983), Temps et récit, tome I, Paris, Éd. du Seuil. (1995), «Le pardon peut-il guérir? », Esprit, 210, p. 77-82. (1990), Soi-même comme un autre, Paris, Seuil. (2000), La mémoire, l'histoire, l'oubli, Paris, Seuil.

Volf, M. (2006), The End of Memory. Remembering Rightly in a Violent World, Michigan / Cambridge, Grand Rapids / Eerdmans.

Von Trier, L. (2003), Dogville, Danemark, 2h57min.

\section{Résumé}

Les auteurs abordent la question du pardon à partir de l'expérience des victimes d'abus sexuels qui la posent souvent d'une façon très aiguë. Le christianisme tient généralement le pardon pour un devoir chrétien, fondé sur une théologie qui considère la réconciliation comme le cœur de l'histoire de salut. Le Nouveau Testament porte un appel à pardonner qui ne peut être nié. Par contre, celui-ci ne va pas nécessairement sans condition ni en faisant abstraction du contexte de la faute ou de la relation. Opérant une étrange inversion de la dynamique du pardon, la psychologie considère de son côté les bienfaits du pardon pour celui ou celle qui le donne plus que pour celui ou celle qui le reçoit. La psychologie connaît aussi les longs cheminements et les impasses du pardon dont la théologie ne saurait faire abstraction. Cela appelle une théologie qui soit plus sensible à la justice et qui approche avec plus de retenue la question du pardon et de la réconciliation. Comme l'amour, le pardon est un don et il ne peut être forcé.

Le pardon n'est pas une obligation dont il faudrait se culpabiliser ou culpabiliser les victimes, souvent les plus faibles, de ne pas y répondre. Si la grâce de Dieu permet de pardonner là où la volonté et le pouvoir humains 
touchent à leurs limites, peut-être y a-t-il aussi une grâce qui permet de retenir le pardon jusqu'à ce que quelque chose change, voire une grâce qui permet de faire justice pour que la vie se poursuive en abondance.

\section{Abstract}

The authors address the issue of forgiveness from the experience of victims of sexual abuse who often bear it very acutely. Christianity generally holds forgiveness as a Christian duty, based on a theology that views reconciliation as the heart of salvation history. The New Testament carries a call to forgive that cannot be denied. But it is not necessarily without conditions, and we should not disregard the context of the fault or the relationship between the actors. In a strange inversion of the dynamics of forgiveness, psychology on the other hand considers the benefits of forgiveness for the one who forgives rather than for the one who is forgiven. It also knows the long paths and dead ends of forgiveness which theology cannot ignore. This calls for a theology that is more sensitive to victims and justice, and that handles the issue of forgiveness and reconciliation more cautiously. Like love, forgiveness is a gift and it cannot be forced.

Forgiveness is not an obligation that should be imposed on victims, who often are the weakest ones. If the grace of God allows us to forgive where human will and power reach their limits, maybe there also is a grace to refuse forgiveness until something changes or a grace to make justice so that life abounds. 